\title{
Análise da Revisão Cochrane: O Papel dos Antiagregantes Plaquetários para Prevenir a Pré- Eclâmpsia e as Suas Complicações. Cochrane Database Syst Rev. 2019;10:CD004659.
}

\author{
Analysis of the Cochrane Review: Antiplatelet Agents \\ for Preventing Pre-Eclampsia and Its Complications. \\ Cochrane Database Syst Rev. 2019;10:CD004659.
}

\author{
Catarina REIS-DE-CARVALHO ${ }^{1}$, Miguel BIGOTTE VIEIRA², João COSTA ${ }^{3,4}$, António VAZ-CARNEIRO ${ }^{3,4}$
} Acta Med Port 2021 Dec;34(12):810-814 - https://doi.org/10.20344/amp.15995

\section{RESUMO}

A pré-eclâmpsia está associada à produção intravascular deficiente de prostaciclina, um vasodilatador, e à produção excessiva de tromboxano, um vasoconstritor e estimulante da agregação plaquetária. Essas observações levaram à hipótese de os antiagregantes plaquetários, em particular o ácido acetilsalicílico, poderem, em dose reduzida, prevenir ou retardar o desenvolvimento da pré-eclâmpsia. Esta revisão da Cochrane teve como objetivo avaliar a eficácia e segurança de antiagregantes plaquetários comparativamente a placebo ou a ausência de tratamento, quando administrados a mulheres em risco de desenvolver pré-eclâmpsia. Foi realizada uma revisão sistemática da literatura até setembro de 2019, através de pesquisa nas seguintes bases de dados: Registo de Ensaios de Gravidez e Parto da Cochrane, ClinicalTrials.gov, Plataforma Internacional de Registo de Ensaios Clínicos da OMS (ICTRP) e listas de referência de estudos recuperados. Foram incluídos 77 ensaios clínicos aleatorizados, incluindo 40249 mulheres grávidas em risco de desenvolver pré-eclâmpsia. Cerca de $80 \%$ destas mulheres foram avaliadas em nove dos 77 ensaios incluídos, sendo que oito destes nove ensaios disponibilizavam dados individuais. $O$ ácido acetilsalićlico foi o antiagregante plaquetário avaliado na maioria dos ensaios, incluindo nos nove ensaios de maior dimensão. A presente revisão concluiu, com qualidade de evidência elevada, que a administração de ácido acetilsalicílico em dose reduzida $(50$ - $150 \mathrm{mg})$ a mulheres grávidas está associada a benefícios, considerados de magnitude pequena a moderada, na redução do risco de pré-eclâmpsia, parto prematuro, feto pequeno para a idade gestacional, e morte fetal ou neonatal. A administração de antiagregantes plaquetários a 1000 mulheres resultou em menos 20 gestações com desfechos adversos graves.

Palavras-chave: Cuidado Pré-Natal; Ensaios Clínicos Controlados Aleatórios como Assunto; Inibidores da Agregação Plaquetária/uso terapêutico; Pré-Eclâmpsia/prevenção e controlo; Pré-Eclâmpsia/tratamento farmacológico

\section{ABSTRACT}

Pre-eclampsia is associated with deficient intravascular production of prostacyclin, a vasodilator, and excessive production of thromboxane, a vasoconstrictor and stimulant of platelet aggregation. These observations led to the hypotheses that antiplatelet agents, low-dose aspirin in particular, might prevent or delay development of pre-eclampsia. This Cochrane review aimed to assess the effectiveness and safety of antiplatelet agents, such as aspirin and dipyridamole, when given to women at risk of developing preeclampsia. A systematic review of literature was carried out by searching the following databases up to September 2019: Cochrane Pregnancy and Childbirth's Trials Register, ClinicalTrials.gov, the WHO International Clinical Trials Registry Platform (ICTRP), and reference lists of retrieved studies. Seventy-seven trials were included, including 40249 women at risk of developing pre-eclampsia. About $80 \%$ of these women were evaluated in nine of the 77 trials included, with eight of these nine trials providing individual data. Interventions were administration of an antiplatelet agent, and comparisons were either placebo or no antiplatelet. The present review provides high-quality evidence that administering low-dose aspirin (50 - $150 \mathrm{mg}$ ) to pregnant women led to small-to-moderate benefits, including reductions in the risk of pre-eclampsia, preterm birth, small-for-gestational age fetus, and fetal or neonatal death. Overall, administering antiplatelet agents to 1000 women led to 20 fewer pregnancies with serious adverse outcomes.

Keywords: Platelet Aggregation Inhibitors/therapeutic use; Pre-Eclampsia/drug therapy; Pre-Eclampsia/prevention \& control; Prenatal Care; Randomized Controlled Trials as Topic

\section{QUESTÃO CLÍNICA}

Qual a eficácia e segurança dos antiagregantes plaquetários, como o ácido acetilsalicílico (AAS), administrados em mulheres grávidas com risco de pré-eclampsia?

\section{OBJETIVOS}

Avaliar a eficácia e segurança dos antiagregantes pla- quetários comparativamente a placebo ou ausência de tratamento quando administrados a mulheres em risco de desenvolver pré-eclâmpsia.

\section{METODOLOGIA}

Esta revisão é uma atualização de uma revisão sistemática anterior. Foi realizada uma pesquisa na literatura

\footnotetext{
1. Departamento de Obstetrícia, Ginecologia e Medicina da Reprodução. Faculdade de Medicina. Universidade de Lisboa. Lisboa. Portugal.

2. Serviço de Nefrologia e Transplantação Renal. Centro Hospitalar Lisboa Norte. Lisboa. Portugal.

3. Centro de Estudos de Medicina Baseada na Evidência. Faculdade de Medicina. Universidade de Lisboa. Lisboa. Portugal.

4. Cochrane Portugal. Lisboa. Portugal.

$\triangle$ Autor correspondente: Catarina Reis-de-Carvalho. catarinareiscarvalho@gmail.com

Recebido: 17 de fevereiro de 2021 - Aceite: 21 de setembro de 2021 - Online issue published: 02 de dezembro de 2021

Copyright @ Ordem dos Médicos 2021
} 
até março de 2018, que foi atualizada em setembro de 2019 nas seguintes bases de dados: Cochrane Pregnancy and Childbirth's, ClinicalTrials.gov, the WHO International Clinical Trials Registry Platform (ICTRP) e em listas de referência de estudos.

Pelo menos dois investigadores selecionaram, verificaram e avaliaram independentemente a qualidade metodológica e o risco de viés dos estudos incluídos de acordo com as orientações descritas no Cochrane Handbook for Systematic Reviews of Interventions.

Relativamente ao tipo de intervenção, foram incluídos todos os estudos aleatorizados que comparavam antiagregantes plaquetários (ácido acetilsalicílico em dose reduzida ou dipiridamol) com placebo ou nenhum agente antiplaquetário. Estudos publicados apenas em formato de resumo foram elegíveis para inclusão se estivessem disponíveis informações suficientes. Não foram identificados e, portanto, não foram incluídos, estudos aleatorizados por cluster ou aleatorizados individualmente. Os estudos quasi-aleatorizados foram excluídos.

Os participantes incluídos eram mulheres grávidas com risco de desenvolver pré-eclâmpsia. Foram estudados os seguintes resultados (outcomes):

- Primários: Desenvolvimento de pré-eclâmpsia, morte fetal e neonatal, parto pré-termo, fetos pequenos para a idade gestacional.

- Secundários: hipertensão gestacional, morte materna, indução de trabalho de parto ou cesariana eletiva, taxa de cesariana, episódios hemorrágicos maternos (descolamento de placenta, hemorragia anteparto ou pós-parto, complicações da analgesia epidural, necessidade de transfusão de sangue), morbilidade materna grave (eclâmpsia, insuficiência hepática ou renal, coagulação intravascular disseminada, alterações visuais, distúrbios psiquiátricos graves), episódios hemorrágicos do recém-nascido, alterações de desenvolvimento na infância, utilização de recursos hospitalares (dias de internamento, cuidados intensivos, etc.).

Foram incorporados dados individuais de participantes sempre que disponíveis nos ensaios, ou dados agregados sempre que os primeiros não estavam disponíveis, a fim de permitir análises de subgrupos com maior robustez. Para resultados binários, foram calculados o risco relativo $(R R)$ e o intervalo de confiança de $95 \%$ (IC), com base na população em intenção de tratar. A qualidade da evidência e a incerteza para cada um dos resultados foi avaliada de acordo com a abordagem GRADE (Grading of Recommendations Assessment, Development and Evaluation).

\section{RESULTADOS}

Setenta e sete estudos (40 249 mulheres e seus bebés) foram incluídos, embora três estudos (relativos a 233 mulheres) não tenham contribuído com dados para a meta-análise. Nove dos ensaios que contribuíram com dados eram de maior dimensão amostral (mais de 1000 participantes), representando $80 \%$ das mulheres recrutadas. Todos os estudos eram ensaios clínicos aleatorizados.
Os estudos foram conduzidos em hospitais-maternidade ou unidades materno-fetais hospitalares de 27 países. Os vários estudos definiram as mulheres em alto risco de pré-eclâmpsia através de diferentes metodologias (apenas fatores maternos e pressão arterial média ou também através de dados ecográficos e bioquímicos).

$\mathrm{O}$ ácido acetilsalicílico em dose reduzida isolada foi a intervenção em todos os grandes ensaios e na maioria dos ensaios em geral. A dose nos grandes ensaios clínicos foi de $50 \mathrm{mg}$ (um ensaio, 1106 mulheres), $60 \mathrm{mg}$ (cinco ensaios, 22322 mulheres), $75 \mathrm{mg}$ (um ensaio, 3697 mulheres), $100 \mathrm{mg}$ (um ensaio, 3294 mulheres) e $150 \mathrm{mg}$ (um ensaio, 1776 mulheres). Em geral, a qualidade metodológica dos estudos incluídos era boa. A maioria dos estudos apresentou um risco de viés baixo ou incerto; os grandes ensaios foram todos classificados como tendo um baixo risco de viés.

A Tabela 1 resume os principais resultados, incluindo a qualidade da evidência para cada resultado.

De entre os mesmos, destaca-se que se verificou, com uma qualidade de evidência elevada, uma redução de $18 \%$ no risco de pré-eclâmpsia em qualquer idade gestacional associado ao uso de antiagregantes plaquetários em muIheres em risco de desenvolver pré-eclâmpsia (prevenção primária). Poucos estudos avaliaram o impacto do AAS como prevenção secundária para a pré-eclâmpsia, por exemplo no caso de hipertensão gestacional. Apesar da análise sugerir uma provável diminuição da proteinúria pré-eclâmpsia nestes casos, a evidência disponível é insuficiente para suportar a utilização dos agentes antiplaquetários na prevenção secundária.

Não foi encontrada evidência de qualidade suficiente para concluir se há diferença no efeito terapêutico conforme o início de administração precoce do ácido acetilsalicílico (menos de 16 semanas gestação) ou tardio (mais de 16 semanas gestação).

Quanto ao efeito da dose do ácido acetilsalicílico, verificou-se acima de tudo redução do risco em ensaios que usaram a dose igual ou superior a $75 \mathrm{mg}$ de AAS. Para mulheres alocadas em grupos com menos de $75 \mathrm{mg}$ de AAS parece haver uma redução ligeira do risco, embora o intervalo de confiança a $95 \%$ inclua a possibilidade de ausência de benefício.

É ainda importante referir que esta revisão concluiu que existe uma redução de $16 \%$ do risco de fetos pequenos para a idade gestacional quando as grávidas recebem tratamento com AAS. Esta redução foi particularmente evidente nas grávidas que iniciaram o tratamento antes das 20 semanas de gestação.

\section{COMENTÁRIOS}

A pré-eclâmpsia é uma doença que afeta $3 \%$ a $5 \%$ das gestações e se caracteriza por pressão arterial elevada e proteinúria. ${ }^{1}$ Está associada a complicações maternas e fetais, incluindo acidente vascular cerebral, eclâmpsia, falência orgânica múltipla, restrição do crescimento fetal, morte intrauterina e trabalho de parto prematuro. 
Tabela 1 - Sumário de resultados, adaptado da versão original

\begin{tabular}{|c|c|c|c|c|c|}
\hline \multirow{3}{*}{ Resultados } & \multicolumn{2}{|c|}{$\begin{array}{l}\text { Riscos comparativos ilustrativos* } \\
\text { (IC } 95 \%)\end{array}$} & \multirow{3}{*}{$\begin{array}{l}\text { Efeito relativo } \\
\text { (IC } 95 \%)\end{array}$} & \multirow{3}{*}{$\begin{array}{l}\text { Número de } \\
\text { participantes } \\
\text { (ensaios) }\end{array}$} & \multirow{3}{*}{$\begin{array}{c}\text { Qualidade } \\
\text { da evidência } \\
\text { (GRADE) }\end{array}$} \\
\hline & Risco assumido & Risco correspondente & & & \\
\hline & $\begin{array}{c}\text { Placebo/ausência de } \\
\text { antiagregantes }\end{array}$ & $\begin{array}{c}\text { Antiagregantes } \\
\text { plaquetários }\end{array}$ & & & \\
\hline $\begin{array}{l}\text { Pré-eclâmpsia } \\
\text { proteinúrica }\end{array}$ & 92 por 1000 & $\begin{array}{c}\text { Menos } 16 \text { por } 1000 \\
(\text { menos } 22 \text { a menos } 11)\end{array}$ & $\begin{array}{c}\text { RR } 0,82 \\
(0,77 \text { a } 0,88)\end{array}$ & $\begin{array}{c}36716 \\
\text { (60 estudos) }\end{array}$ & Elevada \\
\hline $\begin{array}{l}\text { Qualquer morte } \\
\text { (perinatal fetal, } \\
\text { neonatal ou antes da } \\
\text { alta hospitalar) }\end{array}$ & 33 por 1000 & $\begin{array}{c}\text { Menos } 5 \text { por } 1000 \\
(\text { menos } 9 \text { a menos } 1)\end{array}$ & $\begin{array}{c}\text { RR } 0,85 \\
(0,76 \text { a } 0,95)\end{array}$ & $\begin{array}{c}35391 \\
\text { (52 estudos) }\end{array}$ & Elevada \\
\hline $\begin{array}{l}\text { Parto pré-termo (antes } \\
\text { das } 37 \text { semanas) }\end{array}$ & 175 por 1000 & $\begin{array}{l}\text { Menos } 16 \text { por } 1000 \\
(\text { menos } 23 \text { a menos } 9)\end{array}$ & $\begin{array}{c}\text { RR } 0,91 \\
(0,87 \text { a } 0,95)\end{array}$ & $\begin{array}{c}35212 \\
\text { (47 estudos) }\end{array}$ & Elevada \\
\hline $\begin{array}{l}\text { Leve para a idade } \\
\text { gestacional/ Restrição } \\
\text { de crescimento fetal }\end{array}$ & 47 por 1000 & $\begin{array}{c}\text { Menos } 7 \text { por } 1000 \\
\text { (menos } 23 \text { a menos } 9)\end{array}$ & $\begin{array}{c}\text { RR } 0,84 \\
(0,76 \text { a } 0,92)\end{array}$ & $\begin{array}{c}35761 \\
\text { (50 estudos) }\end{array}$ & Elevada \\
\hline $\begin{array}{l}\text { Desfechos obstétricos } \\
\text { graves (composto } \\
\text { incluindo morte } \\
\text { materna, morte fetal, } \\
\text { pre-eclâmpsia, feto } \\
\text { leve para a idade } \\
\text { gestacional ou parto } \\
\text { pré-termo) }\end{array}$ & 197 por 1000 & $\begin{array}{l}\text { Menos } 20 \text { por } 1000 \\
\text { (menos } 20 \text { por menos } 8 \text { ) }\end{array}$ & $\begin{array}{c}\text { RR } 0,90 \\
(0,85 \text { a } 0,96)\end{array}$ & $\begin{array}{c}17382 \\
\text { (13 estudos) }\end{array}$ & Elevada \\
\hline $\begin{array}{l}\text { Hemorragia pós-parto } \\
>500 \mathrm{~mL}\end{array}$ & 143 por 1000 & $\begin{array}{l}\text { Mais } 9 \text { por } 1000 \\
\text { (menos } 0 \text { a mais } 19)\end{array}$ & $\begin{array}{c}\text { RR } 1,06 \\
(1,00 \text { a } 1,12)\end{array}$ & $\begin{array}{c}23769 \\
\text { (19 estudos) }\end{array}$ & Moderada \\
\hline $\begin{array}{l}\text { Descolamento de } \\
\text { placenta }\end{array}$ & 7 por 1000 & $\begin{array}{l}\text { Mais } 2 \text { por } 1000 \\
\text { (menos } 0 \text { a mais } 4)\end{array}$ & $\begin{array}{c}\mathrm{RR} 1,21 \\
(0,95 \text { a } 1,54)\end{array}$ & $\begin{array}{c}30775 \\
\text { (29 estudos) }\end{array}$ & Moderada $^{b}$ \\
\hline
\end{tabular}

* A base para o risco assumido (por exemplo, o risco médio do grupo controlo entre os estudos) é obtida com base no risco assumido no grupo de comparação e o efeito relativo da intervenção (e seu IC 95\%)

IC: intervalo de confiança; RR: risco relativo

Grupo de Trabalho GRADE (níveis de evidência):

- Qualidade elevada: Temos muita confiança de que o verdadeiro efeito seja semelhante ao da estimativa do efeito.

- Qualidade moderada: Temos confiança moderada na estimativa do efeito: o verdadeiro efeito é provavelmente semelhante à estimativa do efeito, mas existe a possibilidade de ser substancialmente diferente.

- Qualidade baixa: A nossa confiança na estimativa do efeito é limitada: o verdadeiro efeito pode ser substancialmente diferente da estimativa do efeito.

- Qualidade muito baixa: Temos muito pouca confiança na estimativa do efeito: o verdadeiro efeito é provavelmente substancialmente diferente da estimativa do efeito.

a: Apesar do efeito estimado não ultrapassar o limite definido para heterogeneidade estatística $\left(I^{2}=40 \%\right)$, foi diminuída em um nível a confiança na evidência devido a preocupações relativas à heterogeneidade clínica entre os estudos na metodologia da medição de perda hemática $(-1)$

b: foi diminuída em um nível a confiança na evidência pela amplitude do intervalo de confiança, que inclui risco apreciável para o grupo dos antiagregantes plaquetários e cruzamento da linha da ausência de efeito $(-1)$.

A fisiopatologia da pré-eclâmpsia tem sido extensamente estudada, mas há o consenso científico de que o início do processo fisiopatológico começa com alterações na normal placentação, em particular invasão trofoblástica anormal das artérias espiraladas maternas. ${ }^{2}$

O rastreio da pré-eclâmpsia é particularmente importante no primeiro trimestre. A abordagem tradicional para identificar mulheres com alto risco de pré-eclâmpsia é avaliada através das características maternas e da história médica. Alguns dos fatores de risco mais importantes a considerar são a idade materna avançada, peso materno aumentado, origem africana ou do sudoeste asiático, história pessoal de hipertensão arterial ou diabetes mellitus, gravidez após técnicas de procriação medicamente assistida e antecedentes de história pessoal de PE. ${ }^{3}$ No entanto, este método apenas identifica $40 \%$ dos casos de PE pré-termo e $35 \%$ dos casos de PE de termo. ${ }^{4}$ Atualmente, recomenda-se que o rastreio de PE no primeiro trimestre seja combinado com a utilização de fatores maternos, pressão arterial média, ín- dice de pulsatilidade das artérias uterinas e biomarcador do soro materno placental growth factor (PLGF) - fator angiogénico da placenta, habitualmente diminuído em casos de pré-eclâmpsia. Este método permite a identificação de $90 \%$ dos casos de PE pré-termo e $45 \%$ dos casos de PE de termo. ${ }^{4}$

O mecanismo de ação através do qual o ácido acetilsalicílico tem efeito na redução da pré-eclâmpsia é complexo e ainda não completamente conhecido. Alguns dos mecanismos envolvem a redução de fatores oxidativos e inflamatórios, promoção de angiogénese, redução da apoptose placentar, impacto positivo na vasoconstrição e agregação placentária maternas e, em geral, a melhoria do processo de placentação. ${ }^{5,6}$ Os mesmos mecanismos explicam o motivo pelo qual o AAS também diminui o número de fetos com diagnóstico de restrição de crescimento fetal, condição largamente associada à placentação anormal.

De notar que, na mesma área de investigação, foi publicado posteriormente a esta revisão da Cochrane (janeiro 
de 2020), um estudo aleatorizado, duplamente cego e controlado com placebo, que incluiu 11976 mulheres. ${ }^{10} \mathrm{O}$ objetivo era avaliar se a administração precoce (entre as seis e as 13 semanas de gestação) de ácido acetilsalicílico em dose reduzida $(81 \mathrm{mg})$ permitia a redução de parto pré-termo em países de baixo e médio rendimento. Verificou-se uma redução de $11 \%$ do risco relativo de parto pré-termo (RR 0,89; IC 95\%: 0,81-0,98) e de 14\% do risco de mortalidade periparto (RR 0,86; IC 95\%: 0,73 - 1,00) e de perda fetal (RR 0,86; IC 95\%: 0,74 - 1,00). Em média, por cada 66 mulheres (IC 95\%: 37 - 308), 126 mulheres (IC 95\%: 63 - 279 827) e 115 mulheres (IC 95\%: 58 - 3180) tratadas, ocorrerá menos um parto pré-termo, uma morte periparto e uma perda fetal, respetivamente. ${ }^{10}$

Estes resultados reforçam o potencial benefício da utilização do ácido acetilsalicílico na obstetrícia em diferentes contextos socioeconómicos e clínicos.

Esta revisão levanta várias questões relevantes para os médicos das especialidades que participam na vigilância da gravidez e para as próprias grávidas:

1. Quem são as mulheres que mais beneficiam com a administração de antiagregantes plaquetários para redução do risco de pré-eclâmpsia?

Não está recomendada a administração de ácido acetilsalicílico em todas as grávidas, mas apenas naquelas em que foi identificado um alto risco de pré-eclâmpsia e onde já foi provado que o AAS tem um efeito benéfico substancial. Assim, é fundamental o rastreio do risco da pré-eclâmpsia entre as 11 e as 13 semanas, com recurso a parâmetros ecográficos e bioquímicos. Quando estes não estão disponíveis, é aceitável realizar-se o rastreio com recurso à história clínica (características demográficas maternas e reconhecimento de fatores de risco) e medição da pressão arterial média materna. ${ }^{7}$

\section{Quando deve ser iniciada a medicação?}

Antes das 16 semanas de gravidez, período que corresponde aos principais processos da placentação. O American College of Obstetricians and Gynecologists (ACOG) advoga que o AAS pode ser iniciado até às 28 semanas. ${ }^{8}$

\section{Qual a dose recomendada?}

Não há consenso. As várias normas de orientação recomendam doses entre os 75 e os $150 \mathrm{mg} / \mathrm{dia}$, tomadas à noite. Doses mais elevadas (nomeadamente $150 \mathrm{mg} / \mathrm{dia}$ ) parecem ser mais eficazes. Esta administração está aconselhada desde o primeiro trimestre até às 36 semanas de gestação. ${ }^{9}$ No entanto, existe alguma controvérsia quanto à vantagem da suspensão do AAS antes do parto, já que não parece associar-se a aumento importante dos riscos, e algumas recomendações vão no sentido de manter esta medicação durante todo o período da gravidez. ${ }^{8}$

\section{CONCLUSÕES E IMPLICAÇÕES NA PRÁTICA CLÍNICA}

- Existe evidência de alta qualidade que o ácido acetilsalicílico em doses baixas, administrado antes das 16 semanas de gestação e no contexto de prevenção primária (mulheres em risco de desenvolver pré-eclâmpsia), reduz o risco de desfechos maternos e neonatais relevantes, incluindo redução de $18 \%$ no risco relativo de desenvolver pré-eclâmpsia; de $9 \%$ dos partos pré-termo; de 15\% de morte fetal ou neonatal; e redução de $10 \%$ de efeitos adversos graves em todas as gestações.

- Não existe evidência de alta qualidade quanto à utilização de outros antiagregantes plaquetários, quanto à utilização de ácido acetilsalicílico no contexto de prevenção secundária, e quanto à introdução tardia de AAS após as 16 semanas de gestação.

- Assim se conclui que em mulheres com risco moderado ou alto de pré-eclampsia deve ser considerada a administração de ácido acetilsalicílico em baixa dose (menos de $75 \mathrm{mg}$ ), se não existirem contraindicações e após discussão do benefício/risco com a grávida.

\section{IMPLICAÇÕES PARA A INVESTIGAÇÃO CIENTÍFICA}

A investigação futura nesta área deve procurar esclarecer os seguintes pontos:

- Quais as mulheres que respondem melhor a dose reduzida de AAS, de modo a direcionar o tratamento;

- Se o início do tratamento depois das 12 semanas traz benefícios adicionais sem aumento dos efeitos adversos;

- Qual a segurança de uma dose mais elevada de ácido acetilsalicílico;

- Se existe benefício dos agentes antiplaquetários na prevenção secundária.

- Se existem outros agentes antiplaquetários, para além do AAS, que podem ter um efeito benéfico.

\section{OBSERVAÇÕES}

A visão expressa neste artigo é a dos autores e não reflete necessariamente as visões e políticas da Cochrane, ou de qualquer outra organização à qual os autores estejam associados.

\section{CONTRIBUTO DOS AUTORES}

CRC: Concepção e desenho do trabalho.

MBV: Concepção e revisão do trabalho.

JC: Contribuição cientifica e revisão crítica,

AVC: Revisão critica e aprovação do manuscrito final.

\section{PROTECÇÃO DE PESSOAS E ANIMAIS}

Os autores declaram que os procedimentos seguidos estavam de acordo com os regulamentos estabelecidos pelos responsáveis da Comissão de Investigação Clínica e Ética e de acordo com a Declaração de Helsínquia da Associação Médica Mundial atualizada em 2013.

\section{CONFIDENCIALIDADE DOS DADOS}

Os autores declaram ter seguido os protocolos do seu centro de trabalho acerca da publicação de dados.

\section{CONFLITOS DE INTERESSE}

Os autores declaram não ter qualquer conflito de interesse relativamente ao presente artigo. 


\section{FONTES DE FINANCIAMENTO}

Não foi utilizada nenhuma bolsa ou subsídio para a realização do trabalho.

\section{REFERÊNCIAS}

1. Duley L, Meher S, Hunter KE, Seidler AL, Askie LM. Antiplatelet agents for preventing pre-eclampsia and its complications. Cochrane Database Syst Rev. 2019:10;CD004659.

2. Pijnenborg R, Vercruysse L, Brosens I. Deep placentation. Best Pract Res Clin Obstet Gynaecol. 2011;25:273-85.

3. Wright D, Syngelaki A, Akolekar R, Poon LC, Nicolaides KH. Competing risks model in screening for preeclampsia by maternal characteristics and medical history. Am J Obstet Gynecol. 2015;213:62.e1-10.

4. O'Gorman N, Wright D, Poon LC, Rolnik DL, Syngelaki A, de Alvarado $M$, et al. Multicenter screening for pre-eclampsia by maternal factors and biomarkers at 11-13 weeks' gestation: comparison with NICE guidelines and ACOG recommendations. Ultrasound Obstet Gynecol. 2017:49:756-60.

5. Dutta S, Kumar S, Hyett J, Salomon C. Molecular targets of aspirin and prevention of preeclampsia and their potential association with circulating extracellular vesicles during pregnancy. Int $\mathrm{J}$ Mol Sci. 2019;20:4370.

6. Rolnik DL, Wright D, Poon LC, Syngelaki A, O'Gorman N, de Paco
Matallana C, et al. ASPRE trial: performance of screening for preterm pre-eclampsia. Ultrasound Obstet Gynecol. 2017;50:492-5.

7. Poon LC, Shennan A, Hyett JA, Kapur A, Hadar E, Divakar H, et al. The International Federation of Gynecology and Obstetrics (FIGO) initiative on pre-eclampsia: a pragmatic guide for first-trimester screening and prevention. Int J Gynaecol Obstet. 2019;145:S1-33. Erratum in: Int J Gynaecol Obstet. 2019;146:390-1.

8. The American College of Obstetricians and Gynecologists Committee. ACOG Opinion No. 743: Low-dose aspirin use during pregnancy. Obstet Gynecol. 2018;132:e44-52.

9. Webster K, Fishburn S, Maresh M, Findlay SC, Chappell LC; Guideline Committee. Diagnosis and management of hypertension in pregnancy: summary of updated NICE guidance. BMJ. 2019;366:I5119.

10. Hoffman MK, Goudar SS, Kodkany BS, Metgud M, Somannavar M, Okitawutshu J, et al. Low-dose aspirin for the prevention of preterm delivery in nulliparous women with a singleton pregnancy (ASPIRIN): a randomised, double-blind, placebo-controlled trial. Lancet. 2020;395:285-93.

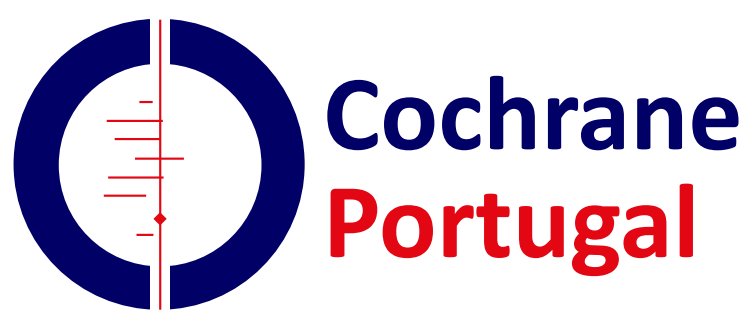

